\title{
Anti-message Logging based Coordinated Checkpointing Protocol for Deterministic Mobile Computing Systems
}

\author{
Praveen Kumar \\ Department of Computer Science \& Engineering, \\ Meerut Institute of Engineering \& Technology, \\ Meerut, India
}

\author{
Ajay Khunteta \\ Singhaniya University \\ Pechri, Rajasthan, India
}

\begin{abstract}
A checkpoint algorithm for mobile computing systems needs to handle many new issues like: mobility, low bandwidth of wireless channels, lack of stable storage on mobile nodes, disconnections, limited battery power and high failure rate of mobile nodes. These issues make traditional checkpointing techniques unsuitable for such environments. Minimum-process coordinated checkpointing is an attractive approach to introduce fault tolerance in mobile distributed systems transparently. This approach is domino-free, requires at most two checkpoints of a process on stable storage, and forces only a minimum number of processes to checkpoint. But, it requires extra synchronization messages, blocking of the underlying computation or taking some useless checkpoints. In this paper, we propose a minimumprocess coordinated checkpointing algorithm for deterministic mobile distributed systems, where no useless checkpoints are taken, no blocking of processes takes place, and anti-messages of very few messages are logged during checkpointing. We try to reduce the loss of checkpointing effort when any process fails to take its checkpoint in coordination with others. We also address the related issues like: failures during checkpointing, disconnections, concurrent initiations of the algorithm.
\end{abstract}

\section{INTRODUCTION}

\subsection{Definitions and Notations}

Checkpoint: Checkpoint is defined as a designated place in a program at which normal process is interrupted specifically to preserve the status information necessary to allow resumption of processing at a later time. A checkpoint is a local state of a process saved on stable storage. By periodically invoking the checkpointing process, one can save the status of a program at regular intervals.

Rollback Recovery: If there is a failure one may restart computation from the last checkpoints thereby avoiding repeating computation from the beginning. The process of resuming computation by rolling back to a saved state is called rollback recovery.

Global State : In a distributed system, since the processes in the system do not share memory, a global state of the system is defined as a set of local states, one from each process. The state of channels corresponding to a global state is the set of messages sent but not yet received.

Orphan Message: A message whose receive event is recorded, but its send event is lost.

Consistent Global State: A global state is said to be "consistent" if it contains no orphan message. To recover from a failure, the system restarts its execution from a previous consistent global state saved on the stable storage during fault-free execution. In distributed systems, checkpointing can be independent, coordinated [3], [8], [11] or quasi-synchronous [2], [9]. Message
Logging is also used for fault tolerance in distributed systems [14].

Asynchronous Checkpointing: Under the asynchronous approach, checkpoints at each process are taken independently without any synchronization among the processes. Because of absence of synchronization, there is no guarantee that a set of local checkpoints taken will be a consistent set of checkpoints. It may require cascaded rollbacks that may lead to the initial state due to domino-effect [7].

Coordinated Checkpointing: In coordinated or synchronous Checkpointing, processes take checkpoints in such a manner that the resulting global state is consistent. Mostly it follows twophase commit structure [3], [8], [11], [22]. In the first phase, processes take tentative checkpoints and in the second phase, these are made permanent. The main advantage is that only one permanent checkpoint and at most one tentative checkpoint is required to be stored. In the case of a fault, processes rollback to the last checkpointed state. Communication-induced Checkpointing: It avoids the domino-effect without requiring all checkpoints to be coordinated [2], [7], [9]. In these protocols, processes take two kinds of checkpoints, local and forced. Local checkpoints can be taken independently, while forced checkpoints are taken to guarantee the eventual progress of the recovery line and to minimize useless checkpoints. As opposed to coordinated checkpointing, these protocols do no exchange any special coordination messages to determine when forced checkpoints should be taken. But, they piggyback protocol specific information [generally checkpoint sequence numbers] on each application message; the receiver then uses this information to decide if it should take a forced checkpoint.

Deterministic Systems: If two processes start in the same state, and both receive the identical sequence of inputs, they will produce the identical sequence outputs and will finish in the same state. The state of a process is thus completely determined by its starting state and by sequence of messages it has received [23, [24], [25].

Checkpoint Interval $(\mathrm{CI})$ : The $\mathrm{i}^{\text {th }} \mathrm{CI}$ of a process denotes all the computation performed between its $i^{\text {th }}$ and $(i+1)^{\text {th }}$ checkpoint, including the $i^{\text {th }}$ checkpoint but not the $(i+1)^{\text {th }}$ checkpoint.

Direct Dependency among Processes: $P_{j}$ is directly dependent upon $P_{k}$ only if there exists $m$ such that $\mathrm{P}_{j}$ receives $m$ from $P_{k}$ in the current $\mathrm{CI}$ and $P_{k}$ has not taken its permanent checkpoint after sending $m$.

Minimum Set: A process $P_{i}$ is in the minimum set only if checkpoint initiator process is transitively dependent upon it.

Minimum-process Coordinated Checkpointing Algorithms : In these algorithms, only a subset of interacting processes (called minimum set) are required to take checkpoints in an initiation.

Anti-Message: David R. Jefferson [29] introduced the concept of anti-message. Anti-message is exactly like an original message in format and content except in one field, its sign. Two messages 
that are identical except for opposite signs are called antimessages of one another. All messages sent explicitly by user programs have a positive (+) sign; and their anti-messages have a negative sign (-). Whenever a message and its anti-message occur in the same queue, they immediately annihilate one another. Thus the result of enqueueing a message may be to shorten the queue by one message rather than lengthen it by one. We depict the antimessage of $\mathrm{m}^{\text {by } \mathrm{m}^{-1}}$.

\subsection{Proposed Work}

In the present study, we propose a minimum-process coordinated Checkpointing algorithm for Checkpointing deterministic distributed applications on mobile systems. We eliminate useless checkpoints as well as blocking of processes during checkpoints at the cost of logging anti-messages of very few messages during Checkpointing. We also try to minimize the loss of checkpointing effort when any process fails to take its checkpoint.

\section{THE PROPOSED CHECKPOINTING ALGORITHM}

\subsection{System Model}

Our system model consists of a number of $\mathrm{MHs}$ which communicate through mobility support stations (MSSs). Each MSS is a fixed network host which provides wireless communication support for a fixed geographical area, called a cell. MSSs are linked together over the wired data networks. The distributed system consisting of n processes, running on MHs or MSSs. The MHs can communicate with the MSS through wireless channels. We assume that wireless channels and logical channels are all FIFO order. If a $\mathrm{MH}$ moves to the cell of another base station, a wireless channel to the old MSS is disconnected and a wireless channel in the new MSS is allocated. However, its checkpoint related information is still with the old MSS. A MH may voluntarily disconnect from mobile computing networks. The $\mathrm{MH}$ does not send and receive any message when it is in a disconnected state. We also assume a closed system that consists of nodes, links, and disks. Input is stored on disk before operation begins. Output is stored on disk when the job ends.

There is no common clock, shared memory or central coordinator. Message passing is the only mode of communication between any pair of processes. The messages originated from a source $\mathrm{Mh}$, are received by the local Mobile support stations and then forwarded to the destination MH. Any process can initiate checkpointing. It is assumed that processes may be failed during processing but there is no communication link failure. Messages are exchanged with finite but arbitrary delays. In our algorithm, we consider that the processes which are running in the distributed mobile systems are deterministic.

\subsection{Basic Idea}

In coordinated checkpointing for mobile distributed systems, there remains a good probability that some process fails to take its checkpoint especially on $\mathrm{MH}$. It can happen due to abrupt disconnection, exhausted battery power, or failure in wireless bandwidth. If a single process fails to take its checkpoint; all the checkpointing effort goes waste, because, each process has to abort its tentative checkpoint. In order to take the tentative checkpoint, an $\mathrm{MH}$ needs to transfer large checkpoint data to its local MSS over wireless channels. Hence, the loss of checkpointing effort may be quite high. Therefore, we propose that in the first phase, all concerned MHs will take mutable checkpoint only. Mutable checkpoint is described in [5], it is stored on the memory of $\mathrm{MH}$ only. In this case, if some process fails to take its checkpoint in the first phase, then other MHs need to abort their mutable checkpoints only. The effort of taking a mutable checkpoint is negligible as compared to the tentative one [5]. When the initiator process comes to know that all relevant processes have taken their mutable checkpoints, it asks all relevant processes to come into the second phase, in which, a process converts its mutable checkpoint into tentative one, i.e. an MH transfers its checkpoint to its local MSS over wireless channel.

During the checkpointing procedure, a process $P_{i}$ may receive $m$ from $P_{j}$ such that $P_{j}$ has taken its tentative checkpoint for the current initiation whereas $P_{i}$ has not. Suppose, $P_{i}$ processes $m$, and it receives checkpoint request later on, and then it takes its checkpoint. In that case, $\mathrm{m}$ will become orphan in the recorded global state. We propose that the anti-messages of only those messages, which can become orphan, should be recorded at the receiver end. In deterministic systems, orphan messages are received as duplicate messages on recovery. A duplicate message is annihilated by its anti-message at the receiver end before processing. Hence, in deterministic distributed systems, an orphan message in global checkpoint does not create any inconsistency during recovery, if its anti-message is logged at the receiver end. By doing so, we avoid the blocking of processes, as well as, the useless checkpoints in minimum-process checkpointing. It should be noted that in minimum-process coordinated checkpointing, some useless checkpoints are taken or blocking of processes takes place. The overheads of logging a few anti-messages may be negligible as compared to taking some useless checkpoints or blocking the processes during checkpointing. This scheme will not be applicable for non-deterministic systems. For nondeterministic systems, we can not deal with an orphan message by logging its anti-message at the receiver end; because, in nondeterministic systems, if we $\log \mathrm{m}_{1}^{-1}$ of orphan message $\mathrm{m}_{1}$, then after recovery, we may get $m_{2}$ in place of $m_{1}$, which may be different from $\mathrm{m}_{1}$. In that case, $\mathrm{m}_{1}^{-1}$ can not annihilate $\mathrm{m}_{2}$, and

$\mathrm{P} 1$

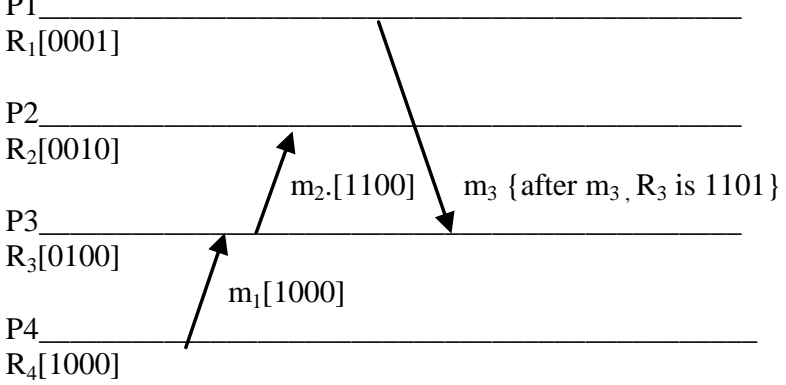

Fig. 2.1

hence the inconsistency will take place. If $\mathrm{P}_{\mathrm{i}}$ become sure that it is not good to take its checkpoint for current initiation or, if $\mathrm{P}_{\mathrm{i}}$ has already taken its checkpoint for the current initiation then it can process $\mathrm{m}$ without any issue. In this case $\mathrm{m}$ can not become orphan.

In the figure $2.1 \mathrm{P}_{4}$ sends $\mathrm{m}_{1}$ to $\mathrm{P}_{3}$ along with its own dependency vector $R_{4}[1000]$. When $P_{3}$ receives $m_{1}$ it update its own dependency vector by taking logical OR of $R_{4} \& R_{3}[0100]$, which comes out to be 1100 . When $\mathrm{P}_{3}$ send $\mathrm{m}_{2}$ to $\mathrm{P}_{2}$, it appends $R 3$ [1100] along with $m_{2}$. When $P_{2}$ receive $m_{2}$, it updates its own dependency vector $R_{2}$ by taking logical OR of $R_{2}$ and $R_{3}$, which 
comes out to be [1110]. In this way, partial transitive dependencies are captured during normal computation. It should be noted that all the transitive dependencies are not captured during normal computation. At time $\mathrm{t} 1$, the dependency vector of $\mathrm{P}_{2}$ shows that $\mathrm{P}_{2}$ is not transitively dependent upon $\mathrm{P}_{1}$, whereas, $\mathrm{P}_{2}$ is transitively dependent upon $\mathrm{P}_{1}$ due to $\mathrm{m}_{3}$ and $\mathrm{m}_{2}$.

\subsection{An Example}

Example: we explain the purposed algorithm with the help of an example. In figure 2.2, at time $\mathrm{t}_{1}, \mathrm{P}_{3}$ initiates checkpointing. At this moment, it dependency vector is[000111]. $\mathrm{P}_{1}$ sends $\mathrm{m}_{1}$ along with its dependency vector [000001]. When $P_{2}$ receives $m$ it update its dependency vector by taking bitwise logical OR of dependency vectors of $\mathrm{P}_{1}$ and $\mathrm{P}_{2}$, which comes out to be [000011]. On receiving $\mathrm{m} 2$ the dependency vector of $\mathrm{P} 3$ become [000111]. At time $t_{1}$, the $\mathrm{P}_{3}$ computes tentative minimum $\operatorname{set}\left\{\mathrm{P}_{1}, \mathrm{P}_{2}, \mathrm{P}_{3}\right\}$ and sends the mutable checkpoint request $\mathrm{w}$ P1 \& P2 and takes its own mutable checkpoint. For an $\mathrm{MH}$ the mutable checkpoint is stored on the disk of $\mathrm{MH}$. After taking its mutable checkpoint, $\mathrm{P}_{3}$ sends $\mathrm{m}_{4}$ to $\mathrm{P}_{4} . \mathrm{P}_{3}$ also piggyback its own csn along with $\mathrm{m}_{4}$. When $\mathrm{P}_{4}$ receive $\mathrm{m}_{4}$, it finds that csn of $\mathrm{P}_{3}$ at the time of sending $\mathrm{m}_{4}$ is 1 ; therefore, $\mathrm{P}_{4}$ concludes that $\mathrm{P}_{3}$ has taken its checkpoint for the new initiation and $\mathrm{P}_{4}$ has not taken checkpoint for the same. Therefore, $\mathrm{P}_{4} \operatorname{logs} \mathrm{m}_{4}^{-1}$ and process $\mathrm{m}_{4}$. when $\mathrm{P}_{2}$ takes its mutable checkpoint $\mathrm{C}_{21}$, it finds that it is dependent upon $\mathrm{P}_{4}$, due to $\mathrm{m}_{3}$ and $\mathrm{P}_{4}$ is not in the tentative minimum set $\left\{\mathrm{P}_{1}, \mathrm{P}_{2}, \mathrm{P}_{3}\right\}$,

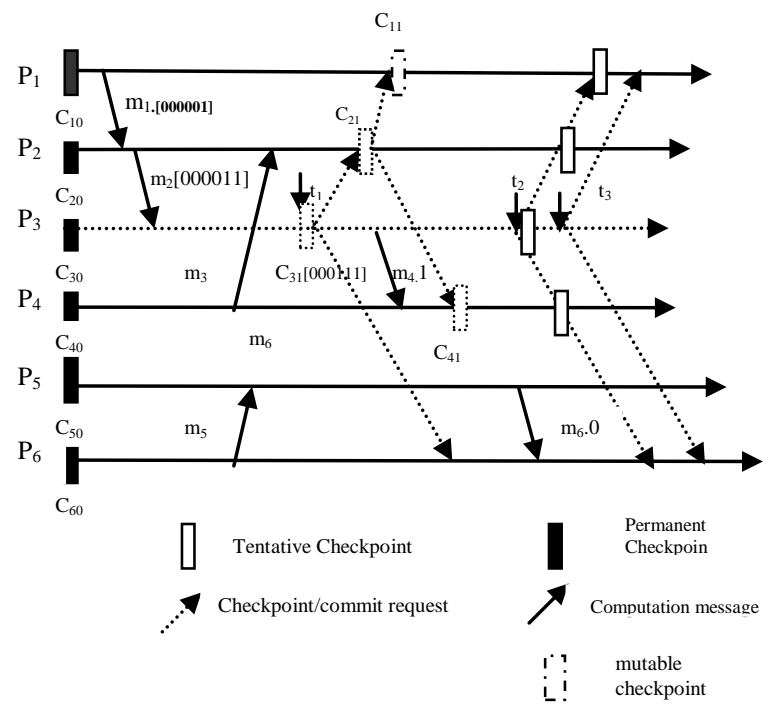

Figure 2.2

therefore, $\mathrm{P}_{2}$ sends checkpoint request to $\mathrm{P}_{4}$ and $\mathrm{P}_{4}$ takes its mutable checkpoint $\mathrm{C}_{41} \cdot \mathrm{P}_{5}$ sends $\mathrm{m}_{6}$ to $\mathrm{P}_{6}$. on the basis of piggybacked csn of $\mathrm{P}_{5}$, on $\mathrm{m}_{6}, \mathrm{P}_{6}$ concludes that $\mathrm{P}_{5}$ has not taken it checkpoint for the current initiation, therefore $\mathrm{P} 6$ process $\mathrm{m} 6$ without logging $\mathrm{m}_{6}{ }^{-1}$. At time $\mathrm{t}_{2}, \mathrm{P}_{3}$ receives response form all process in the minimum set(not shown in the figute) and finds that they have taken their mutable checkpoints successfully, therefore $\mathrm{P}_{3}$ issues tentative checkpoint request to all processes. On getting tentative checkpoint request, a process converts it mutable checkpoint into tentative one and sends the response to initiator process $P_{3}$. At time $t_{3}, P_{3}$ receives responses form the process in minimum set and finds that they have taken their tentative checkpoints successfully, therefore, $\mathrm{P}_{3}$ issues commit request to all process. A process in the minimum set converts its tentative checkpoint into permanent checkpoint and discards it old permanent checkpoint if any. The anti messages are also logged on stable storage. In the present case the global checkpoint is collected as follows: $\left\{\mathrm{C}_{11}, \mathrm{C}_{21}, \mathrm{C}_{31}, \mathrm{C}_{41}, \mathrm{~m}_{4}{ }^{-1}, \mathrm{C}_{50}, \mathrm{C}_{60}\right)$. If the system recovers from this state after a fault, $\mathrm{P}_{3}$ will send $\mathrm{m}_{4}$ again as the processes are assumed to be deterministic. When $\mathrm{P}_{4}$ will receive $m_{4}$, it will find that $m_{4}^{-1}$ is logged at $P_{4} . P_{4}$ concludes that it has already received $\mathrm{m}_{4}$, therefore $\mathrm{P}_{4}$ ignores $\mathrm{m}_{4}$.

\subsection{Data Structures}

Here, we describe the data structures used in the proposed checkpointing protocol. A process on $\mathrm{MH}$ that initiates checkpointing, is called initiator process and its local MSS is called initiator MSS. If the initiator process is on an MSS, then the MSS is the initiator MSS. All data structures are initialized on completion of a checkpointing process, if not mentioned explicitly.

Pr_csn $n_{i}$ : A monotonically increasing integer checkpoint sequence number for each process. It is incremented by 1 on mutable checkpoint.

td_vect $_{\mathrm{i}}$ []: It is a bit array of length $\mathrm{n}$ for $\mathrm{n}$ process in the system. td_vect $\left[\right.$ [ ] $=1$ implies $\mathrm{P}_{\mathrm{i}}$ is transitively dependent upon $\mathrm{P}_{\mathrm{j}}$. When $\mathrm{P}_{\mathrm{i}}$ receives $m$ from $P_{j}$ such that $P_{j}$ has not taken any permanent checkpoint after sending $m$ then $P_{i}$ sets td_vect $t_{i}[j]=1$. When $P_{i}$ commit its checkpoint, it sets $t_{-} \_v e c t_{i}[]=0$ for all processes except for itself which is initialized to 1 .

chkpt-st $t_{\mathrm{i}}$ : A boolean which is set to ' 1 ' when $\mathrm{P}_{\mathrm{i}}$ takes a tentative checkpoint; on commit or abort, it is reset to zero

m_vect[]: An bit array of size $\mathrm{n}$ for $\mathrm{n}$ processes in the systems. When $\mathrm{P}_{\mathrm{i}}$ starts checkpointing procedures, it computes tentative minimum set as follows: $\mathrm{m}_{-} \operatorname{vect}[\mathrm{j}]=\mathrm{td}_{-} \operatorname{vect}_{\mathrm{i}}[\mathrm{j}]$ where $\mathrm{j}=1,2$, ....,n.

TC[]: An array of size $\mathrm{n}$ to save information about the processes which have taken their tentative checkpoints in the second phase. When process $P_{j}$ takes its tentative checkpoint then $j^{\text {th }}$ bit of this vector is set to 1 . It is initialized to all zeros in the beginning of the checkpointing process. It is maintained by the checkpoint initiator MSS only.

MC[]: A bit array of size $n$, maintained by initiator MSS. MC[i]=1 implies $\mathrm{P}_{\mathrm{i}}$ has taken its mutable checkpoint in the first phase.

MSS_chk_taken2[]: A bit array of length $\mathrm{n}$ maintained by each MSS. MSS_chk_taken2[i] $=1$ implies $\mathrm{P}_{\mathrm{i}}$ has taken its tantative checkpoint successfully in the second phase.

MSS_chk_request2[]: A bit array of length $\mathrm{n}$ at each MSS. MSS_chk_request2[i] =1, Pi has been issued tentative checkpoint request in the second phase.

Max_time: it is a flag used to provide timing in checkpointing operation. It is initialized to zero when timer is set and becomes ' 1 ' when maximum allowable time for collecting global checkpoint expires.

MSS_plist[]: A bit array of length $\mathrm{n}$ for $\mathrm{n}$ processes which is maintained at each MSS MSS_plist ${ }_{K}[j]=1$ implies each process $P_{j}$ is running on $\mathrm{MSS}_{\mathrm{k}}$. If $\mathrm{P}_{\mathrm{j}}$ is disconnected, then it checkpoint related information is on $\mathrm{MSS}_{\mathrm{k}}$.

MSS_chk_taken: A bit array of length $\mathrm{n}$ bits maintained by the MSS. MSS_chk_taken $[j]=1$ implies $P_{j}$ which is in the cell of MSS has taken its mutable checkpoint in the first phase.

MSS_chk_request: A bit array of length $n$ at each MSS. The $j^{\text {th }}$ bit of this array is set to ' 1 ' whenever initiator sends the checkpoint request to $P_{j}$ and $P_{j}$ is in the cell of this MSS. 
MSS_fail_bit: A flag maintained on every MSS, initialized to ' 0 '; set to ' 1 ' when any process in the cell of MSS fails to take tentative checkpoint

$\mathrm{P}_{\mathrm{in}}$ : The process which has initiated the checkpointing operation MSS $_{\text {in }}$ : The MSS which has $P_{\text {in }}$ in its cell

$\mathrm{p}_{\text {_csn }}$ in : checkpoint sequence number of initiator process

g_chkpt: A flag which indicates that some global checkpoint is being saved

csn[]: An array of size $n$, maintained on every MSS, for $n$ processes. $\operatorname{csn}[\mathrm{i}]$ represens the most recently committed checkpoint sequence number of $\mathrm{P}_{\mathrm{i}}$. After the commit operation, if $m_{-}$vect[i] $=1$ then $\operatorname{csn}[\mathrm{i}]$ is incremented. It should be noted that entries in this array are updated only after converting tentative checkpoints in to permanent checkpoints and not after taking tentative checkpoints.

m_vect1[]: An array of size $n$ maintained on every MSS. It contains those new processes which are found on getting checkpoint request from initiator.

m_vect2[]: An array of size $n$. for all $\mathrm{j}$ such that m_vect1[j] $\neq 0$,

m_vect $2=m_{-}$vect $2 \bigcup$ m_vect 1 .

m_vect3[]: An array of length $\mathrm{n}$; on receiving $\mathrm{m}_{-}$vect3[], m_vect[], m_vect1[] along with checkpoint request [c_req] or on the computation of $m_{-}$vect1[] locally: m_vect3[]=m_vect3[] $\cup$

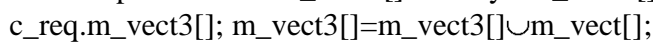

m_vect3[]=m_vect3[] $\cup$ c_req.m_vect1[]; m_vect3[] $=$ m_vect3[] $\cup$ m_vect1[]; m_vect3[] maintains the best local knowledge of the minimum set at an MSS;

\subsection{Computation of m_vect[], m_vect1[], m_vect2[], m_vect3[]:}

1. Suppose a process $P_{r}$ wants to initiate checkpointing procedure. Its send its request to its local MSS, say $\mathrm{MSS}_{\mathrm{r} . .} \mathrm{MSS}_{\mathrm{r}}$ maintains the dependency vector of $\mathrm{P}_{\mathrm{r}}$ (say td_vect $\mathrm{r}_{\mathrm{r}}[\mathrm{]}) \cdot \mathrm{MSS}_{\mathrm{r}}$ coordinates checkpointing on behalf of $\mathrm{P}_{\mathrm{r}}$. It computes tentative minimum set as follows:

$$
\forall{ }_{\mathrm{i}=1, \mathrm{n}} \text { m_vect }[\mathrm{i}]=\mathrm{td}_{-} \operatorname{vect}_{\mathrm{r}}[\mathrm{i}]
$$

2. On receving m_vect[] from $\mathrm{MSS}_{\mathrm{r}}$, any $\mathrm{MSS}$ (say $\mathrm{MSS}_{\mathrm{S}}$ ) computes the m_vect1[] as follows:

Suppose MSSs maintains the process $\mathrm{P}_{\mathrm{j}}$ such that $\mathrm{P}_{\mathrm{j}} \in \mathrm{MSSs}$ and $\mathrm{P}_{\mathrm{j}} \in \mathrm{m}_{\text {_vect }}$

$\forall i, m_{-} \mathrm{vect}_{1}[\mathrm{i}]=1$ iff $\mathrm{m}_{-} \operatorname{vect}[\mathrm{i}]=0$ and $\mathrm{td}_{-} \operatorname{vect}_{\mathrm{j}}[\mathrm{i}]=1$

m_vect1[] maintains the new processes found for the minimum set when a process receives the checkpoint request.

m_vect $2=\mathrm{m} \_$vect $2 \mathrm{U}$ m_vect 1

$\forall$ i, m_vect $1[\mathrm{i}]=0$

3. $\mathrm{m}_{-}$vect $3=\mathrm{m} \_$vect $\mathrm{U}$ m_vect 2

$\mathrm{MSS}_{\text {in }}$ sends c_req to $\mathrm{MSS}_{\mathrm{s}}$ along with $\mathrm{m}_{-}$vect[] and some process (say $\mathrm{P}_{\mathrm{k}}$ ) is found at $\mathrm{MSS}_{\mathrm{s}}$, which takes the checkpoint to this c_req. All MSSs maintains the processes of minimum set to the best of their knowledge in m_vect3. It is required to minimize duplicate checkpoint requests. Suppose, there exists some process (say $\mathrm{P}_{1}$ ) such that $\mathrm{P}_{k}$ is directly dependent upon $\mathrm{P}_{1}$ and $\mathrm{P}_{1}$ is not in the $m_{-}$vect 3 , then $\mathrm{MSS}_{\mathrm{s}}$ sends c_req to $\mathrm{P}_{\mathrm{l}}$. The new processes found for the minimum set while executing a potential checkpoint request at an MSS are stored in m_vect1. When an MSS finds that all the local processes, which were asked to take checkpoints, have taken their checkpoints, it sends the response to the $\mathrm{MSS}_{\text {in }}$ along with $\mathrm{m}_{-}$vect2; so that $\mathrm{MSS}_{\text {in }}$ may update its knowledge about minimum set and wait for the new processes before sending commit. In this way, $\mathrm{MSS}_{\text {in }}$ sends commit only if all the processes in the minimum set have taken their tentative checkpoints.

\subsection{The Checkpointing Protocol}

As the wireless bandwidth is a scarce commodity in mobile systems; therefore; we impose minimum burdon on wireless channels. The local MSS of an MH acts on behalf of the process running on $\mathrm{MH}$. We piggyback checkpoint sequence numbers and dependency vectors onto normal computation messages, but this information is not sent on wireless channels. The local MSS of an $\mathrm{MH}$, strips all the additional information from the computation message and sends it to the concerned MH. The dependency vector of a process running on an $\mathrm{MH}$ is maintained by its local MSS.

Our algorithm is distributed in nature in the sense that any process can initiate checkpointing. If two processes initiate checkpointing concurrently, then the checkpoint imitator of the lower process ID will prevail. The local MSS of a process coordinates checkpointing on its behalf. Suppose two processes $P_{i}$ and $P_{j}$ starts checkpointing concurrently and $\mathrm{MSS}_{\mathrm{p}}$ and $\mathrm{MSS}_{\mathrm{q}}$ are their local MSS respectively then $\mathrm{MSS}_{\mathrm{p}}$ and $\mathrm{MSS}_{\mathrm{q}}$ will send checkpoint requests along with tentative minimum set to all the MSS's. MSSp will receive the checkpoint request of MMSq and MMSq will receive the checkpoint request of MSSp. Suppose Process-ID of $P_{i}$ is less than Process-ID of $P_{j}$, then the checkpoint initiates of $P_{i}$ will prevail. Any other MSS will automatically ignore the request of $\mathrm{P}_{\mathrm{j}}$ because, every MSS will compare the process id of $\mathrm{P}_{\mathrm{i}}$ and $P_{j}$.

We propose that any process in the system can initiate the checkpointing operation. When a process $P_{\text {in }}$ starts checkpointing procedure, it send its request to its local MSS say $\mathrm{MSS}_{\text {in }} . \mathrm{MSS}_{\text {in }}$ computes the tentative minimum set (m_vect[]) as follows:

$\forall$ i=1,n m_vect[i] $=$ td_vect[i]. MSS $_{\text {in }}$ coordinates checkpointing process on behalf of $\mathrm{P}_{\mathrm{in}}$. We want to emphasize that td_vect ${ }_{\text {in }}[]$ contains the processes on which $\mathrm{P}_{\text {in }}$ transitively depends and the set is not complete.

$\mathrm{MSS}_{\text {in }}$ sends c-req (mutable checkpoint request) to all MSS's along with $\mathrm{m}_{-}$vect $_{\text {in }}[]$. When an MSS say MSS $_{\mathrm{p}}$ receives c-req; it sends the c-req to all such process which are running in its cell and are also the member of $m_{-}$vect $_{\text {in }}[]$. Suppose, $P_{j}$ gets the checkpoint request at $\mathrm{MSS}_{\mathrm{p}}$. Now we find any process $\mathrm{P}_{\mathrm{k}}$ such that $\mathrm{P}_{\mathrm{k}}$ does not belong to $\mathrm{m}_{-} \mathrm{vect}_{\mathrm{in}}[]$ and $\mathrm{P}_{\mathrm{k}}$ belongs to td_vect $\mathrm{j}[]$. In this case, $P_{k}$ is also included in the minimum set. During checkpointing, suppose $\mathrm{P}_{\mathrm{i}}$ takes it checkpoint and after that it send $m$ to $P_{j}$ such that $P_{j}$ has not taken its checkpoint at the time of receiving $m$. If $P_{j}$ receive $m$ and it gets checkpoint request later on then $\mathrm{m}$ will become orphan. In order to handle this situation, we $\log \mathrm{m}^{-1}$ at $\mathrm{P}_{\mathrm{j}}$. if $\mathrm{P}_{\mathrm{j}}$ takes the checkpoint, then $\mathrm{m}$ becomes the orphan message in the recorded global state. In deterministic system, orphan message will be delivered again at receiving and it will be annihilated by its anti-message stored at the receiver end. Suppose, $P_{i}$ sends $m$ to $P_{j}$ and $P_{j}$ has already logged $m^{-1}$. it implies $P_{j}$ has already received $m$ and $m$ is a duplicate message, therefore, $\mathrm{P}_{\mathrm{j}}$ ignores $\mathrm{m}$ and also delete $\mathrm{m}^{-1}$.

For a disconnected $\mathrm{MH}$ that is a member of minimum set, the MSS that has its disconnected checkpoint, considers its disconnected checkpoint as the required come. When a MSS learns that its concerned processes in its cell have taken their mutable checkpoints, it sends the response to $\mathrm{MSS}_{\text {in }}$. It should be noted that in the first phase, all processes take the mutable 
checkpoints. For a process running on a static host, mutable checkpoint is equivalent to tentative checkpoint. But, for an $\mathrm{MH}$, mutable checkpoint is different from tentative checkpoint. In order to take a tentative checkpoint, an $\mathrm{MH}$ has to record its local state and has to transfer it to its local MSS. But, the mutable checkpoint is stored on the local disk of the MH. It should be noted that the effort of taking a mutable checkpoint is very small as compared to the tentative one[5]. When the initiator MSS comes to know that all processes in the minimum set taken their mutable checkpoint successfully $\mathrm{MSS}_{\text {in }}$ issues tentative checkpoint request to all MSSs along with the exact minimum set. Alternatively, if $\mathrm{MSS}_{\text {in }}$ comes to know that some process has failed to take its checkpoint in the first phase, it issues abort request to all MSS. In this way the MHs need to abort only the mutable checkpoints, and not the tentative ones. In this way we try to reduce the loss of checkpointing effort in case of abort of checkpointing algorithm in first phase.

When an MSSs receives the tentative checkpoint request, it asks all relevant process to convert their mutable checkpoints into tentative ones. In order to convert its mutable checkpoint into tentative one, an $\mathrm{MH}$ needs to transfer its checkpoint data to its local MSS. A process on static node has to do nothing in order to convert its mutable checkpoint into tentative one. When an MSS learns that all its relevant processes have taken their tentative checkpoint, it informs $\mathrm{MSS}_{\text {in. }}$ Finally, when the initiator MSS learns that all processes in the minimum set have taken their tentative checkpoints successfully, it issues commit request to all MSSs. When a process in the minimum set gets the commit request, it converts it tentative checkpoint into permanent one and discards its earlier permanent checkpoint, if any. All the antimessages are also stored on stable storage along with committed checkpoints.

\section{PERFORMANCE EVALUATION}

In [13], initiator process/MSS collects dependency vectors for all the processes and computes the minimum set and sends the checkpointing request to all the processes with minimum set. The algorithm is non-blocking; the message received during checkpointing may add processes to the minimum set. It suffers from additional message overhead of sending request to all processes to send their dependency vectors and all processes send dependency vectors to the initiator process. But in our algorithm, no such overhead is imposed. The Cao-Singhal [5] suffers from the formation of checkpointing tree as shown in basic idea. In our algorithm, theoretically, we can say that the length of the checkpointing tree will be considerably low as compared to algorithm [5], as most of the transitive dependencies are captured during the normal processing. We do not compare our algorithm with Prakash-Singhal [15], as Cao-Singhal proved that there no such algorithm exists [4].

Furthermore, in [5] algorithm, transitive dependencies are captured by direct dependencies. Hence the average number of useless checkpoints requests will be significantly higher than the proposed algorithm. In [5], huge data structure are piggybacked along with checkpointing request, because they are unable to maintain exact dependencies among processes. Incorrect dependencies are solved by these huge data structures. In our case, no such data structures are piggybacked on checkpointing request and no such useless checkpoint requests are sent., because we are able to maintain exact dependencies among processes and furthermore, are able to capture transitive dependencies during normal computation at the cost of piggybacking bit vector of length $\mathrm{n}$ for $\mathrm{n}$ processes.

In Cao-Singhal algorithm[5], some useless checkpoints are taken or some blocking of processes takes place, we avoid both by logging anti-messages of very few message at the receiver end only during the checkpoint process. The drawback of our algorithm is that it is not applicable for non deterministic systems while the CS[5] algorithm is designed for non deterministic systems. Our algorithm is distributed in nature that any process can initiate checkpointing. We do not allow concurrent executions of the protocol. If we allow, concurrent executions then our goal of minimizing checkpointing efforts will be defeated, many processes will start taking checkpoint quite frequently without advancing their recovery line significantly.

\section{CONCLUSION}

In this paper, we have proposed a minimum-process non-intrusive checkpointing protocol for deterministic mobile distributed systems, where no useless checkpoints are taken. The number of processes that take checkpoints is minimized to 1) avoid awakening of MHs in doze mode of operation, 2) minimize thrashing of MHs with checkpointing activity, 3) save limited battery life of MHs and low bandwidth of wireless channels. In minimum-process checkpointing protocols, some useless checkpoints are taken or blocking of processes takes place; we eliminate both by logging anti-messages of very few selective messages at the receiver end only during the checkpointing period. The overheads of logging a few anti-messages may be negligible as compared to taking some useless checkpoints or blocking the processes during checkpointing. We try to reduce the checkpointing time by avoiding checkpointing tree which may be formed in Cao-Singhal [5] algorithm. We captured the transitive dependencies during the normal execution by piggybacking dependency vectors onto computation messages. The Z-dependencies are well taken care of in this protocol. We also avoided collecting dependency vectors of all processes to find the minimum set as in [4], [13].

\section{REFERENCES}

[1]. Acharya A. and Badrinath B. R., "Checkpointing Distributed Applications on Mobile Computers," Proceedings of the $3^{\text {rd }}$ International Conference on Parallel and Distributed Information Systems, pp. 73-80, September 1994.

[2]. Baldoni R., Hélary J-M., Mostefaoui A. and Raynal M., "A Communication-Induced Checkpointing Protocol that Ensures Rollback-Dependency Trackability," Proceedings of the International Symposium on Fault-Tolerant-Computing Systems, pp. 68-77, June 1997.

[3]. Cao G. and Singhal M., "On coordinated checkpointing in Distributed Systems", IEEE Transactions on Parallel and Distributed Systems, vol. 9, no.12, pp. 1213-1225, Dec 1998.

[4]. Cao G. and Singhal M., "On the Impossibility of Min-process Non-blocking Checkpointing and an Efficient Checkpointing Algorithm for Mobile Computing Systems," Proceedings of International Conference on Parallel Processing, pp. 37-44, August 1998.

[5]. Cao G. and Singhal M., "Mutable Checkpoints: A New Checkpointing Approach for Mobile Computing systems," IEEE Transaction On Parallel and Distributed Systems, vol. 12, no. 2, pp. 157-172, February 2001. 
[6]. Chandy K. M. and Lamport L., "Distributed Snapshots: Determining Global State of Distributed Systems," ACM Transaction on Computing Systems, vol. 3, No. 1, pp. 63-75, February 1985.

[7]. Elnozahy E.N., Alvisi L., Wang Y.M. and Johnson D.B., "A Survey of Rollback-Recovery Protocols in Message-Passing Systems," ACM Computing Surveys, vol. 34, no. 3, pp. 375-408, 2002.

[8]. Elnozahy E.N., Johnson D.B. and Zwaenepoel W., "The Performance of Consistent Checkpointing," Proceedings of the $11^{\text {th }}$ Symposium on Reliable Distributed Systems, pp. 39-47, October 1992.

[9]. Hélary J. M., Mostefaoui A. and Raynal M., "Communication-Induced Determination of Consistent Snapshots," Proceedings of the $28^{\text {th }}$ International Symposium on Fault-Tolerant Computing, pp. 208-217, June 1998.

[10]. Higaki H. and Takizawa M., "Checkpoint-recovery Protocol for Reliable Mobile Systems," Trans. of Information processing Japan, vol. 40, no.1, pp. 236-244, Jan. 1999.

[11]. Koo R. and Toueg S., "Checkpointing and Roll-Back Recovery for Distributed Systems," IEEE Trans. on Software Engineering, vol. 13, no. 1, pp. 23-31, January 1987.

[12]. Neves N. and Fuchs W. K., "Adaptive Recovery for Mobile Environments," Communications of the ACM, vol. 40, no. 1, pp. 68-74, January 1997.

[13] Parveen Kumar, Lalit Kumar, R K Chauhan, V K Gupta “A Non-Intrusive Minimum Process Synchronous Checkpointing Protocol for Mobile Distributed Systems" Proceedings of IEEE ICPWC-2005, pp 491-95, January 2005.

[14]. Pradhan D.K., Krishana P.P. and Vaidya N.H., "Recovery in Mobile Wireless Environment: Design and Trade-off Analysis," Proceedings $26^{\text {th }}$ International Symposium on Fault-Tolerant Computing, pp. 16-25, 1996.

[15]. Prakash R. and Singhal M., "Low-Cost Checkpointing and Failure Recovery in Mobile Computing Systems," IEEE Transaction On Parallel and Distributed Systems, vol. 7, no. 10, pp. 1035-1048, October1996.

[16]. Ssu K.F., Yao B., Fuchs W.K. and Neves N. F., “Adaptive Checkpointing with Storage Management for Mobile Environments," IEEE Transactions on Reliability, vol. 48, no. 4, pp. 315-324, December 1999.

[17]. J.L. Kim, T. Park, "An efficient Protocol for checkpointing Recovery in Distributed Systems," IEEE Trans. Parallel and Distributed Systems, pp. 955-960, Aug. 1993.
[18]. L. Kumar, M. Misra, R.C. Joshi, "Checkpointing in Distributed Computing Systems" Book Chapter "Concurrency in Dependable Computing", pp. 273-92, 2002.

[19]. L. Kumar, M. Misra, R.C. Joshi, "Low overhead optimal checkpointing for mobile distributed systems" Proceedings. 19th IEEE International Conference on Data Engineering, pp 686 - 88, 2003.

[20]. Ni, W., S. Vrbsky and S. Ray, "Pitfalls in Distributed Nonblocking Checkpointing", Journal of Interconnection Networks, Vol. 1 No. 5, pp. 47-78, March 2004.

[21]. L. Lamport, "Time, clocks and ordering of events in a distributed system" Comm. ACM, vol.21, no.7, pp. 558-565, July 1978.

[22]. Silva, L.M. and J.G. Silva, "Global checkpointing for distributed programs", Proc. $11^{\text {th }}$ symp. Reliable Distributed Systems, pp. 155-62, Oct. 1992.

[23]. Parveen Kumar, Lalit Kumar, R K Chauhan, "A Nonintrusive Hybrid Synchronous Checkpointing Protocol for Mobile Systems", IETE Journal of Research, Vol. 52 No. 2\&3, 2006.

[24]. Parveen Kumar, "A Low-Cost Hybrid Coordinated Checkpointing Protocol for mobile distributed systems", Mobile Information Systems. pp 13-32, Vol. 4, No. 1, 2007.

[25]. Lalit Kumar Awasthi, P.Kumar, "A Synchronous Checkpointing Protocol for Mobile Distributed Systems: Probabilistic Approach" International Journal of Information and Computer Security, Vol.1, No.3 pp 298-314.

[26]. Johnson, D.B., Zwaenepoel, W., " Sender-based message logging", In Proceedingss of $17^{\text {th }}$ international Symposium on Fault-Tolerant Computing, pp 14-19, 1987.

[27]. Johnson, D.B., Zwaenepoel, W., "Recovery in Distributed Systems using optimistic message logging and checkpointing. pp 171-181, 1988.

[28] Pushpendra Singh, Gilbert Cabillic, "A Checkpointing Algorithm for Mobile Computing Environment", LNCS, No. 2775, pp 65-74, 2003.

[29] David R. Jefferson, "Virtual Time", ACM Transactions on Programming Languages and Systems, Vol. 7, NO.3, pp 404-425, July 1985 .

[30] Sunil Kumar, R K Chauhan, Parveen Kumar, “A Minimumprocess Coordinated Checkpointing Protocol for Mobile Computing Systems", International Journal of Foundations of Computer science, Vol 19, No. 4, pp 1015-1038 (2008). 